\title{
Computational Models of the Human Body for Medical Image Analysis
}

\author{
Nicholas Ayache \\ Research Director \\ Asclepios Project-Team \\ INRIA \\ 2004 Route des Lucioles, \\ 06902, Sophia-Antipolis, France
}

\begin{abstract}
Medical image analysis brings about a collection of powerful new tools designed to better assist the clinical diagnosis and to model, simulate, and guide more efficiently the patient's therapy. A new discipline has emerged in computer science, closely related to others like computer vision, computer graphics, artificial intelligence and robotics.

In this talk, I describe the increasing role of computational models of anatomy and physiology to guide the interpretation of complex series of medical images, and illustrate my presentation with applications to cardiac and brain diseases. I conclude with some promising trends, including the analysis of in vivo confocal microscopic images.

N. Ayache, O. Clatz, H. Delingette, G. Malandain, X. Pennec, and M. Sermesant. Asclepios: a Research Project at INRIA for the Analysis and Simulation of Biomedical Images. In Proceedings of the Colloquium in Memory of Gilles Kahn, LNCS, 2007. Springer. Note: In press (20 pages).
\end{abstract}

This reference and others are available at http://www-sop.inria.fr/asclepios/ 